\title{
Análisis fisiológico de las tareas de entrenamiento en fútbol sala
}

http://dx.doi.org/10.11606/1807-5509201800010049

\author{
Carlos LAGO-FUENTES ${ }^{* \text { *** }}$ \\ Antonio BORES-CEREZAL ** \\ Martín LÓPEZ-NIETO ${ }^{* * *}$ \\ Adrián PAZ-FRANCO* \\ Marcos MECÍAS-CALVO**** \\ Cristian ABELAIRAS-GÓMEZ* \\ Roberto BARCALA-FURELOS* \\ Ezequiel REY-EIRAS*
}

* Universidad de Vigo,

Pontevedra, Espanha.

${ }^{* *}$ Universidad

Europea Del Atlántico,

Santander, Espanha.

*** Palma Futsal,

Espanha.

\section{Resumen}

El objetivo del estudio fue obtener un perfil condicional de las tareas en fútbol sala, analizándolas en función de 5 variables (tiempo de intervención, duración, FCMáx, FCMedia y concentración de lactato). Participaron 8 jugadores profesionales, con una muestra total de 70 tareas agrupadas en 8 subcategorias. El análisis estadístico fue realizado con el SPSS 20.0, y consta de análisis descriptivos generales y dos pruebas ANOVA de un factor con corrección de Bonferroni. Los resultados muestran que las tareas de juego real no alcanzan la carga fisiológica de la competición. Además, las tareas de resistencia a la velocidad alcanzan una lactacidemia superior al resto. Finalmente, las tareas de transición, movilidad, campo completo, $4 \times 4$ y portero-jugador tienen características condicionales similares, adecuadas para el desarrollo de la resistencia mixta y umbral anaeróbico. Se concluye que las tareas analizadas sirven para el desarrollo de las diferentes vías metabólicas caracteristicas del fútbol sala.

Palabras Clave: FC Máxima; FC Media; Lactato; Competición; Entrenamiento.

\section{Introducción}

La capacidad de rendimiento en los deportes colectivos se ve afectada por numerosos factores y aspectos, no sólo los factores físico-condicionales, sino también los factores técnico-coordinativos, táctico-decisionales y psico-sociales ${ }^{1}$. Siendo conscientes de que lo que el jugador manifiesta en la competición es, en gran parte, el resultado de los efectos de las tareas realizadas en los entrenamientos, es imprescindible considerar el entrenamiento y la competición como dos elementos indisolubles interdependientes. Por tanto, el entrenamiento exigirá el conocimiento más exacto posible de las exigencias impuestas por la competición.

El fútbol sala es un deporte en donde se enfrentan 2 equipos de 5 jugadores cada uno, con un máximo de 7 suplentes, excepto en torneos FIFA o UEFA en los cuales se permite un máximo de 9 suplentes, sobre una pista de 40x $20 \mathrm{~m}$, con porterías de 2 metros de alto por 3 de ancho, cuyo objetivo es meter más goles que el rival en la portería contraria. Cada partido está compuesto por 2 partes de 20 minutos a tiempo parado, con un descanso entre partes de 10 minutos. Así, la duración total de los partidos de la Liga Nacional de Fútbol Sala (LNFS) está entre los 75-80 minutos, 40 minutos de tiempo real y 35 minutos de pausas que se producen durante el juego ${ }^{2-4}$.

A lo largo de un partido de fútbol sala se realizan una media de 274 acciones, las cuales la mayor parte tienen una duración corta, entre 0 y 10s, y una duración media de pausa entre acciones de $12,2 \mathrm{~s}^{4}$, por lo que el ratio actividad - pausa se establece en 1:1,45. En consecuencia, el jugador/a tendrá que sufrir grandes adaptaciones a esfuerzos intermitentes de máxima intensidad, factor a tener en cuenta a la hora de planificar el entrenamiento. Teniendo esto en cuenta, a la hora de buscar modelos de planificación que se ajusten a esta realidad, se han tenido que rechazar todos aquellos modelos de planificación tradicionales basados en el control de los elementos entrenables de manera aislada. Esto se debe a su incompatibilidad con la aplicación integrada de las cargas de entrenamiento en plazos de tiempo muy cortos, ya que los jugadores 
tienen que competir y estar a un alto nivel cada 4-7 días durante 10 meses. Los modelos tradicionales no se ajustan a esta realidad ya que necesitan un periodo preparatorio mucho más amplio con el fin de alcanzar el pico de forma del deportista ${ }^{6}$, algo que no se da en los deportes colectivos ya que tienen un máximo de 6-8 semanas de pretemporada.

Asimismo, con el modelo de microestructuración de Seirulo, la planificación de las cargas de entrenamiento está supeditada bajo el modelo integrado, que se define como la preparación concurrente física-técnica-táctica en el contexto que intervienen con la competición ${ }^{6}$. Es decir, en la actualidad, se busca un diseño de tareas que engloben el trabajo de capacidades técnicas, tácticas, psicológicas y condicionales de manera conjunta, aumentando la eficiencia en el entrenamiento y buscando la mayor transferencia posible a las situaciones que se dan en la competición.

Para conseguir esto, es muy importante conocer la carga de cada tarea de entrenamiento. En las tareas integradas esto adquiere mayor dificultad, ya que implica que hay que darle un valor a cada una de las tareas, teniendo en cuenta el volumen, la intensidad, la densidad, la complejidad, los elementos técnicotácticos presentes, el momento de la temporada en que se lleva a cabo, etc ${ }^{7}$.

Hay una gran cantidad de estudios que se están centrando en el control de la carga del entrenamiento, debido a que todavía hay muchas dudas sobre si estas tareas cumplen el objetivo condicional para poder adquirir el estado de forma esperado, siendo escasos los artículos de investigación que analizan las características de los esfuerzos de entrenamiento en el fútbol sala ${ }^{7-10}$.

Basándonos en lo expuesto anteriormente, el objetivo principal del estudio fue realizar un análisis de los parámetros fisiológicos de las tareas integradas o globales del entrenamiento de un equipo profesional de fútbol sala, basándonos en el tiempo de intervención, duración de la tarea, frecuencia cardiaca media, frecuencia cardiaca máxima y lactacidemia al finalizar la tarea. El segundo objetivo de la presente investigación consistió en intentar definir los parámetros fisiológicos alcanzados en tareas de simulación de juego real.

\section{Método}

\section{Muestra}

Ocho jugadores profesionales, que ocupan puestos de campo (se excluyeron a los jugadores del equipo filial y a los porteros), compiten en Primera División de la LNFS, que realizan una media de 6 entrenamientos por semana, más un partido de competición el fin de semana. Antes del inicio del estudio, todos los jugadores fueron informados del procedimiento a seguir y firmaron el consentimiento informado para la realización del estudio. El estudio fue aprobado por la Comisión Ética de Investigación de la Universidad de Vigo, así como cumple con los requisitos marcados por la Declaración de Helsinki para investigaciones experimentales. Se analizaron un total de 70 tareas.

Las características de los jugadores analizados son de 24,2 \pm 4,2 años de edad, 75,6 $\pm 8,4 \mathrm{~kg}$ de masa, $178,1 \pm 9,4 \mathrm{~cm}$ de altura, $10,0 \pm 0,89 \%$ graso $y$ con 3,03 $\pm 2,8$ años de experiencia de media en Primera División.

\section{Material}

Para realizar la recogida de los datos de las diversas variables analizadas se han empleado el Pulsómetro Polar Team 2 (Polar Team System, Polar Electro, Kempele, Finlandia) con registros de frecuencia cardiaca (FC) cada segundo, para controlar todos los latidos de los jugadores a analizar. Todos los jugadores estaban familiarizados con el uso de los pulsómetros con bandas torácicas, ya que trabajan con ellos desde principio de temporada y los usan diariamente. El software Polar Precision Performance SW, para valorar las FC medias (FCMedia) y máximas (FCMáx) de cada jugador en todas las tareas analizadas. Para analizar el lactato post-tarea se empleó el Lactate Scout (SensLab GmbH, Leipzig, Alemania). Por otro lado, para registrar los tiempos de cada tarea se emplearon los cronómetros digitales (Kalenji 100 y CasioW215), y una hoja de registro para recoger todos los datos obtenidos. 


\section{Procedimiento}

El diseño utilizado fue una investigación cuasiexperimental, en el que se han contemplado dos factores: el primero, el tipo de tarea y el segundo, las variables técnico/fisiológicas.

El estudio se realizó a mediados de temporada durante el periodo competitivo, tomando los datos durante 11 entrenamientos, 10 tácticos y 1 condicional, considerándose entrenamientos tácticos aquellos en los que se realizaban tareas aplicadas al juego y, condicional, aquél en el cual se trabajaban aspectos condicionales a través de medios analíticos aislados del juego, analizándose un total de 70 tareas. Las tareas forman parte de la metodología habitual de entrenamiento de estos jugadores por lo que su aprendizaje y dominio está adquirido. Un grupo de discusión de técnicos de nivel mundial formado por seleccionadores nacionales y preparadores físicos, comprobaron y participaron en la construcción de las tareas propuestas, verificando su viabilidad para los objetivos propuestos. El personal médico del club supervisó y participó en la recogida de los datos.

Los tipos de tareas que fueron analizadas en la presente investigación se basaron en su objetivo principal, definiéndolas como:

- Transiciones: tareas en $40 \times 20 \mathrm{~m}$ con objetivo de trabajar inferioridades defensivas buscando asimetrías durante la tarea, con diferentes pautas.

- Movilidad: tareas de 5x5 en 28x20m con la finalidad de trabajar movilidad grupal durante el juego, buscando dualidades, juego de 4, $1 \times 1$ en banda, etc.

- Estrategia: tareas de 5x5 en 20x20m, con el objetivo de trabajar las jugadas a balón parado cada vez que saliese el balón.

- Resistencia a la velocidad: tareas analíticas específicas en circuito con el objetivo de trabajar la capacidad láctica. Se realizaron 4 series entre 60 y $90 \mathrm{seg}$, con un ratio de trabajo descanso de 1:4.

- Campo completo: tareas de 5x5 en 40x20m.

- 4x4: tareas de 4x4 en 28x20m enfrentándose 4 contra 4 , con objetivo principal el entrenamiento de la defensa presionante.

- Portero-jugador: tareas de 5x5 en 40x20m para trabajar el portero-jugador con resultado favorable siempre a la defensa.

- Juego real: tareas de 5x5 en 40x20m con entorno de competición (cronómetro a tiempo parado, árbitro y normas oficiales).

En cada una de las tareas fueron observadas variables relacionadas con la carga interna, como son la FCMáx y FCMedia de cada, así como el lactato, tomado entre 1 y 2 minutos tras finalizar la tarea. La FCMáx fue considerada el punto más alto registrado por el jugador durante la tarea, así como la FCMedia fue el promedio de sus registros durante toda la tarea.

Además, fueron controlados los tiempos de duración de cada tarea, así como el tiempo de intervención de cada jugador, ya que a lo largo del ejercicio había cambios por lo que no se correspondía con el tiempo total.

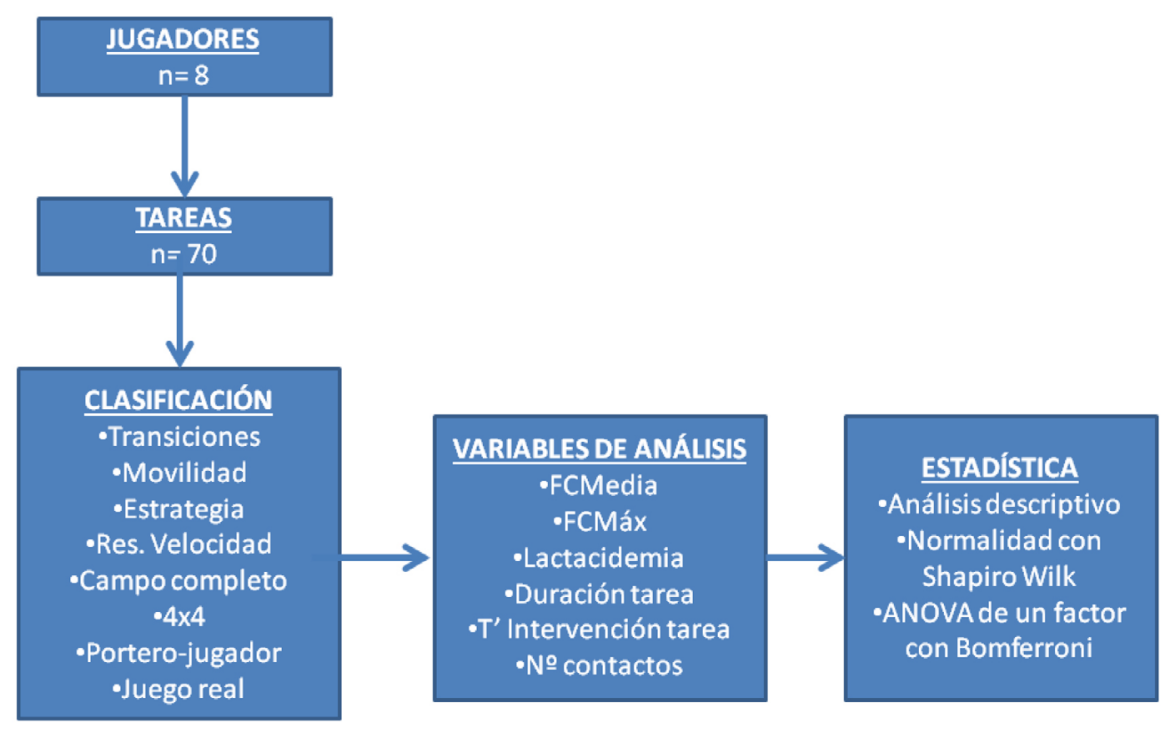

FIGURA 1 - Esquema de investigación. 


\section{Estadística}

Inicialmente fue aplicado el test de Shapiro-Wilk para comprobar si la distribución de la muestra era normal. Para el análisis descriptivo de los datos se han utilizado las medidas de tendencias central (media) y de dispersión (desviación típica). Para comprobar el efecto de cada factor, así como el de la interacción entre ellos, se utilizó el ANOVA con el test de Bonferroni. Se calculó también la magnitud de las diferencias entre los distintos tipos de tareas de acuerdo al tamaño del efecto de Cohen (TE). El criterio para interpretar la magnitud del TE fue: $<0,2$ trivial, 0,2 a 0,6 pequeña, 0,6 a 1,2 moderada $y>1,2$ grande $^{11}$. Los análisis estadísticos se realizaron con el programa SPSS para Windows, versión 20.0 (SPSS Inc., IBM, USA) estableciendo como nivel de significación $\mathrm{p}<0,05$.

\section{Resultados}

En la TABLA 1, se exponen los datos de cada variable en relación a cada uno de los tipos de tareas con el fin de obtener un perfil medio de las mismas, pudiéndose observar cómo varían las medias y desviaciones típicas. Entre los datos expuestos destacan, en relación a la duración y tiempo de intervención, las tareas de resistencia a la velocidad, ya que son las más cortas, así como juego real la de mayor duración.

Con respecto a las variables fisiológicas, la FCMáx superior pertenece a las tareas de juego real $(179,63 \pm 7,71 \mathrm{lat} / \mathrm{min})$, así como la inferior a estrategia $(159,3 \pm 6,45 \mathrm{lat} / \mathrm{min})$. En relación a la FCMedia, los datos más bajos pertenecen también a las tareas de estrategia $(130,9 \pm 6,1 \mathrm{lat} / \mathrm{min})$, siendo los valores más elevados en resistencia a la velocidad $(154,88 \pm 5,36 \mathrm{lat} / \mathrm{min})$ y no en juego real. Finalmente, la concentración de lactato más elevada pertenece, de nuevo, a las tareas de resistencia a la velocidad $(9,28 \pm 2,89 \mathrm{mmol} / \mathrm{L})$, y la más reducida, a estrategia $(2,18 \pm 0,78 \mathrm{mmol} / \mathrm{L})$.

TABLA 1 - Análisis descriptivo de los tipos de tareas.

a Duración en

Segundos

${ }^{b}$ FCMáx y FCMed

en lat/min

${ }^{c}$ La en $\mathrm{mmol} / \mathrm{L}$

\begin{tabular}{lccccccccccc}
\hline \multirow{2}{*}{ Tipo tarea } & \multicolumn{2}{c}{ Duración $^{a}$} & \multicolumn{3}{c}{$\begin{array}{c}\text { Tiempo } \\
\text { intervención }\end{array}$} & \multicolumn{2}{c}{ Fcmáx $^{b}$} & \multicolumn{2}{c}{ Fcmedia $^{b}$} & \multicolumn{2}{c}{ Lactato $^{c}$} \\
\cline { 2 - 11 } & Media & DT & Media & DT & Media & DT & Media & DT & Media & DT \\
\hline 1. Transiciones & 411,33 & 125,29 & 212,17 & 92,24 & 169,33 & 10,80 & 140,00 & 11,88 & 6,23 & 1,99 \\
2. Movilidad & 468,65 & 40,92 & 317,82 & 39,64 & 174,06 & 8,93 & 151,12 & 9,23 & 4,63 & 1,83 \\
3. Estrategia & 565,20 & 56,12 & 325,60 & 85,37 & 159,30 & 6,45 & 130,90 & 6,10 & 2,18 & 0,78 \\
4. Resistencia Velocidad & 76,50 & 2,45 & 46,50 & 2,45 & 167,25 & 4,59 & 154,88 & 5,36 & 9,28 & 2,89 \\
5. Campo completo & 436,30 & 95,70 & 331,40 & 99,99 & 167,30 & 12,03 & 143,30 & 13,95 & 3,63 & 1,21 \\
6. 4x4 & 456,33 & 113,99 & 208,83 & 83,01 & 174,00 & 5,69 & 143,83 & 6,65 & 4,92 & 2,62 \\
7. Portero-jugador & 303,60 & 38,11 & 288,20 & 45,33 & 167,2 & 7,92 & 148,20 & 8,50 & 4,54 & 1,82 \\
8. Juego real & 905,00 & 424,98 & 557,00 & 212,82 & 179,63 & 7,71 & 150,88 & 6,85 & 6,11 & 2,32 \\
\hline
\end{tabular}

En la TABLA 2, se presentan los resultados de la prueba ANOVA con corrección de Bonferroni, donde se realiza una comparación entre las 5 variables analizadas con respecto a los diferentes tipos de tareas.

En relación a la variable duración, las tareas de resistencia a la velocidad tienen diferencias significativas en relación con todas las tareas, ya que es la más corta. A su vez, la tarea de portero-jugador presenta duraciones mayores en relación con las tareas de movilidad, estrategia y campo completo, así como las tareas de estrategia, también presentan una duración mayor, de manera significativa, en relación a las tareas de movilidad y campo completo. 
TABLA 2 - Diferencias significativas entre tareas según las variables estudiadas.

\begin{tabular}{|c|c|c|c|c|c|}
\hline \multicolumn{3}{|c|}{ Duración } & \multicolumn{3}{|c|}{ Tiempo de intervención } \\
\hline & Tarea & TE & & Tarea & TE \\
\hline \multirow{7}{*}{$\begin{array}{l}\text { Resistencia } \\
\text { velocidad } \\
\text { (menor) }\end{array}$} & Transición* & grande & \multirow{6}{*}{$\begin{array}{l}\text { Resistencia } \\
\text { Velocidad } \\
\text { (menor) }\end{array}$} & Movilidad*** & grande \\
\hline & Movilidad*** & grande & & Estrategia $^{* * *}$ & grande \\
\hline & Estrategia*** $^{* *}$ & grande & & Campo Completo*** & grande \\
\hline & Campo Completo*** & grande & & $4 \times 4^{*}$ & grande \\
\hline & $4 \times 4^{* *}$ & grande & & Portero-jugador** & grande \\
\hline & Portero-jugador** & grande & & Juego real** & grande \\
\hline & Juego real* & grande & \multirow{2}{*}{$\begin{array}{l}\text { Juego real } \\
\text { (mayor) }\end{array}$} & Transición* & grande \\
\hline \multirow{3}{*}{$\begin{array}{l}\text { Portero- } \\
\text {-jugador } \\
\text { (menor) }\end{array}$} & Movilidad** $^{* *}$ & moderado & & $4 \times 4^{*}$ & grande \\
\hline & Estrategia** $^{* *}$ & grande & & FCMáx & \\
\hline & Campo Completo* & grande & \multirow{4}{*}{$\begin{array}{l}\text { Estrategia } \\
\text { (menor) }\end{array}$} & Tarea & TE \\
\hline \multirow{3}{*}{$\begin{array}{l}\text { Estrategia } \\
\text { (mayor) }\end{array}$} & Movilidad* & grande & & Movilidad** & grande \\
\hline & Campo Completo* & grande & & $4 \times 4^{*}$ & grande \\
\hline & Lactacidemia & & & Juego real ${ }^{* * *}$ & grande \\
\hline \multirow{3}{*}{$\begin{array}{l}\text { Estrategia } \\
\text { (menor) }\end{array}$} & Transición* & grande & & FCMedia & \\
\hline & Movilidad** & grande & \multirow{4}{*}{$\begin{array}{l}\text { Estrategia } \\
\text { (menor) }\end{array}$} & Movilidad $^{* * *}$ & grande \\
\hline & Resistencia Velocidad** $^{* *}$ & grande & & $\begin{array}{l}\text { Resistencia Veloci- } \\
\text { dad }^{* * *}\end{array}$ & grande \\
\hline \multirow{2}{*}{$\begin{array}{l}\text { Resistencia } \\
\text { Velocidad } \\
\text { (mayor) }\end{array}$} & Movilidad** & grande & & Portero-jugador** & grande \\
\hline & Campo Completo** $^{* *}$ & grande & & Juego real** & grande \\
\hline
\end{tabular}

${ }^{*}<0,05 ;{ }^{* *}<0,005$

$* * *<0,001 / \mathrm{TE}$.

Tamaño del efecto
Respecto a la variable del tiempo de intervención de los jugadores, observamos que las tareas de resistencia a la velocidad registran una gran diferencia significativa con el resto de tareas, a excepción de las tareas de transición, ya que muestran una media de 46,5s. A su vez, el juego real presenta diferencias significativas en relación con las transiciones y los ejercicios de $4 \times 4$ en $28 \times 20$, siendo la tarea de juego real la de mayor tiempo de intervención.

Por otro lado, observamos que únicamente se encuentran diferencias significativas entre las FC máximas de las tareas de estrategia en relación con las tareas de movilidad, $4 \times 4$ y juego real, siendo mayor la FCMáx de estas últimas.

A su vez, las diferencias encontradas respecto a la variable FCMedia no son mucho mayores, y vuelven a relacionar la menor FCMedia en tareas de estrategia con la mayor FCMedia de las tareas de movilidad, resistencia a la velocidad, porterojugador y juego real.
De esta manera, las tareas de estrategia son las que mayor diferencia significativa demuestran con respecto al resto de tipo de tareas, en base a su FCMáx y FCMedia.

Por último, respecto a la última variable, la lactacidemia, se encuentran diferencias significativas entre las bajas concentraciones de lactato en las tareas de estrategia con las mayores concentraciones en las tareas de transición, movilidad y, resistencia a la velocidad.

Las tareas de resistencia a la velocidad presentan significación en relación a su mayor concentración de lactato con las tareas de movilidad y campo completo.

La FIGURA 2 muestra las concentraciones de lactato a través de un diagrama de cajas, en el que se observa que en 6 de las 8 tareas analizadas (Transiciones, Movilidad, Resistencia a la Velocidad, $4 \mathrm{x} 4$, Portero Jugador y Juego Real) se trabaja por encima del umbral anaeróbico teórico, es decir, por encima de los $4,0 \mathrm{mmol} / \mathrm{L}$. 
*: Diferencias significativas $(P$ valor $<0,05)$ con respecto a Transición, Movilidad y Res. Velocidad; \#: diferencias significativas $(P$ valor $<0,05)$ con respecto a Movilidad y Campo completo

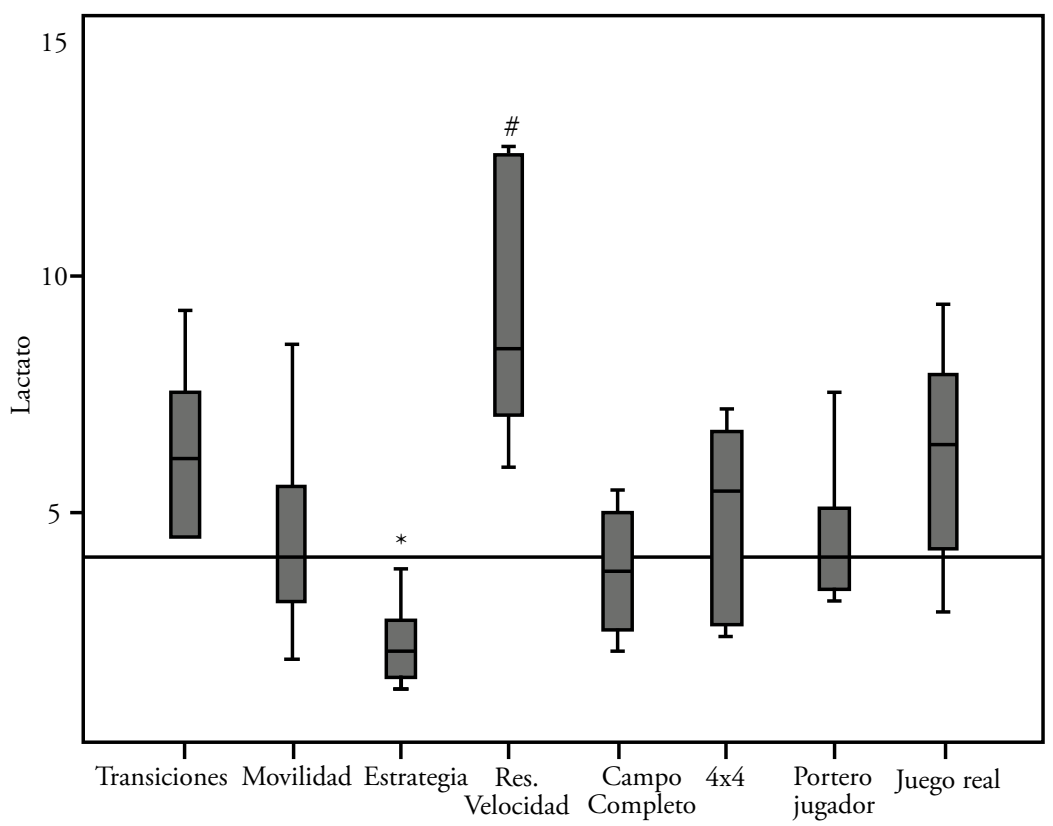

FIGURA 2 - Diagrama de cajas de $\{\mathrm{La}\}$ respecto al tipo de tarea.

\section{Discusión}

El objetivo principal del estudio fue realizar un análisis de los parámetros fisiológicos de las tareas integradas o globales del entrenamiento de un equipo profesional de fútbol sala, basándonos en diferentes variables fisiológicas y temporales. Además, vamos a considerar si son adecuadas para alcanzar las características fisiológicas del fútbol sala, como es principalmente la resistencia mixta ${ }^{12}$.

Las tareas de estrategia, que obtuvieron $130 \pm 6,1 \mathrm{lat} / \mathrm{min}$ como frecuencia cardiaca media, por debajo del 70\% FCMáx, se quedan bastante alejadas de la FCMedia de competición $(174 \mathrm{lat} / \mathrm{min})^{5}$. Por tanto, se pueden emplear con el fin de trabajar la capacidad aeróbica desde un punto de vista fisiológico, básica en los períodos preparatorios para empezar a construir el estado de forma del jugador, o como tarea regenerativa. Esto no quiere decir que su componente táctico pueda llegar a provocar otro tipo de adaptaciones en el organismo.

La FCMedia obtenida en las tareas de transiciones, movilidad, campo completo, $4 \times 4$, portero-jugador y juego real, está entre 143 y 154 lat $/$ min (75-80\% FCMáx). A pesar de no alcanzar la FCMedia de competición, no se quedan muy alejadas de otros estudios ${ }^{2,13,14}$, en los que obtuvieron como FCMedia entre 160-170 lat/min. Por lo tanto pueden ser empleadas para el desarrollo de la resistencia mixta, característica fisiológica más importante en el fútbol sala ${ }^{12}$.

Las tareas de resistencia a la velocidad, con respecto a su FCMedia, podría interpretarse que sirven para desarrollar también la potencia aeróbica, ya que entra en el mismo rango que el grupo anteriormente citado. Esto sería cierto, si no fuese porque su concentración de lactato media supera los $9 \mathrm{mmol} / \mathrm{L}$, lo que supone una tarea tipo para el desarrollo de la resistencia específica, con estímulos anaeróbicos ${ }^{15}$ más intensos que el resto de tareas. Estas tareas se corresponden con las concentraciones de lactato en competición ${ }^{3}$, por tanto pueden ser empleadas para el desarrollo de la tolerancia a la acumulación de lactato.

Continuando con el análisis de las tareas en función de su concentración de lactato, encontramos que las tareas de transiciones y juego real sirven para trabajar de manera más efectiva la resistencia específica por encima del umbral anaeróbico ${ }^{15}$, ya que se alcanzan más de $6 \mathrm{mmol} / \mathrm{L}$ de lactacidemia, superando así el umbral anaeróbico, tradicionalmente marcado en $4 \mathrm{mmol} / \mathrm{L}$.

En este sentido, las tareas de movilidad, campo completo, $4 \times 4$ y portero-jugador, debido a que sus lactacidemia se encuentra entre 4 y $5 \mathrm{mmol} / \mathrm{L}$, pueden ser empleadas para el entrenamiento del considerado umbral anaeróbico ${ }^{16,17}$ con el objetivo de trabajar 
de manera más específica la resistencia mixta. Estas tareas las encajaríamos un escalón por debajo de las tareas ya citadas, ya que la lactacidemia es inferior.

Finalmente, las tareas de estrategia, como ya se especificó anteriormente, no alcanzan una carga condicional alta, manteniéndose en valores muy bajos, con $2 \mathrm{mmol} / \mathrm{L}$ de lactato, de ahí que se pueda emplear como tarea regenerativa o recuperadora.

Considerando otros estudios realizados sobre el análisis condicional de diferentes tipos de tareas de entrenamiento, se observa que la FCMedia obtenida en las tareas integradas de 4x4 fue de 143,83 $\pm 6,65$ lat $/ \mathrm{min}$, un 75\% FCMáx, lo que supone un dato menor que el $81,5 \pm 10,6 \%$ FCMáx $^{17}$. Estas diferencias también pueden ser debidas a que los jugadores empleados como muestra eran semiprofesionales ${ }^{18}$, mientras que la muestra empleada para el presente estudio, eran todos profesionales.

Por otro lado, analizando la FCMedia en las tareas de juego real, mediante las cuales se intenta reproducir las características de un partido competitivo, los datos obtenidos en el presente estudio, 150,88 $\pm 6,58 \mathrm{lat} / \mathrm{min}$, se asemejan con la FCMedia obtenida en tareas de juego real con $155 \pm 13,9 \mathrm{lat} / \mathrm{min}^{8}$, y ambas son menores que la FCMedia del jugador de fútbol sala en competición, que se encuentra entre 160 y $170 \mathrm{lat} / \mathrm{min}^{2,13,14}$. Estas diferencias se pueden explicar debido a que, aunque en el entrenamiento se intente simular el contexto "competición" del modo más real posible, no deja de ser una tarea más de entrenamiento, por tanto el estado de activación del jugador y de implicación, no llega a ser del mismo alcance.

Esto mismo se justifica con las concentraciones de lactato obtenidas. En el presente estudio, las tareas de juego real obtuvieron una lactacidemia media de $6,11 \pm 2,32 \mathrm{mmol} / \mathrm{L}$, similares a $5,2 \pm 1,08 \mathrm{mmol} / \mathrm{L}^{8}$ y $5,5 \pm 1,4 \mathrm{mmol} / \mathrm{L}^{19}$ vuelven a ser inferiores frente a los $9 \mathrm{mmol} / \mathrm{L}^{3,20}$. Aun así, este dato de lactacidemia en competición es muy relativo ya que no se conoce el tiempo de esfuerzo que realizaron los jugadores en los 5 últimos minutos de partido, que son los que realmente influyen en la concentración de lactato post partido.

Con respecto al análisis de las tareas en función de las diferencias significativas encontradas entre ellas a partir de cada una de las variables estudiadas en este trabajo, en la variable FCMáx sólo se han encontrado diferencias significativas comparando las tareas de estrategia con las tareas de movilidad, 4x4 y juego real. Esto puede deberse a que las tareas de estrategia requieren mayor exigencia a nivel cognitivo, con mayor número de correcciones e interrupciones. Por el contrario, las tareas de movilidad, $4 \mathrm{x} 4 \mathrm{y}$ juego real, lo que buscan es provocar ante un ritmo de juego alto, con acciones continuadas, con el fin de reproducir en el entrenamiento situaciones de juego que se dan en la competición. Respecto al resto de grupos no se encontraron diferencias significativas.

En relación a la FCMedia, encontramos también diferencias significativas de las tareas de estrategia con movilidad, resistencia a la velocidad, porterojugador y juego real. Continuando con el argumento anteriormente expresado, esto se explica porque los grupos con los que hay diferencias son los que tienen mayor similitud con la competición, principalmente portero-jugador, movilidad y juego real. Por otro lado, la resistencia a la velocidad, es una tarea condicional analítica de corta duración, a máxima intensidad, por lo que la FC media es significativamente más alta.

En relación a la concentración de La, volvemos a encontrar diferencias significativas entre las tareas de estrategia con transiciones, movilidad, resistencia a la velocidad y juego real. Esto se explica debido a las características anteriormente citadas de las tareas de estrategia y del resto de tareas, esto es, que las últimas nombradas se caracterizan por generar un alto ritmo de juego, con cambios de ritmo propios del fútbol sala al intentar simular las acciones más comunes en dicho deporte, como son las acciones técnicotácticas grupales, las transiciones ofensivas y defensivas y el trabajo en metabolismo láctico.

Por otro lado, las tareas de resistencia a la velocidad reflejan también diferencias en relación a las tareas de movilidad y campo completo, ya que son las tareas, junto con estrategia, que muestran concentraciones de lactato más distantes con respecto a las demás, las primeras por tener mayor concentración de lactato y las segundas, por tener la más baja.

Centrándonos ahora en el aspecto temporal, pasamos a analizar la duración. Las tareas de resistencia a la velocidad muestran diferencias significativas con todas las demás. Esto es debido a que es una tarea analítica condicional que busca como objetivo acumular lactato, por tanto, es corta a máxima intensidad.

Dentro de la duración, el portero-jugador tiene diferencias significativas con movilidad, estrategia y campo completo, durando menos el $5 \times 4$ que las otras previamente mencionadas. Esto puede deberse al hecho de que éste se suele emplear al final de las sesiones, $y$ se busca gran concentración en menos tiempo, que el resto de tareas que tienen como finalidad trabajar más aspectos del juego, y pueden durar más. 
Las tareas de estrategia demuestran que duran más que las tareas de movilidad y campo completo. Esto puede deberse a que las primeras se busca trabajar el mayor número de jugadas a balón parado posibles, con continuos matices por parte de los entrenadores, lo que hace que se prolonguen en el tiempo.

Finalmente, analizando el tiempo de intervención, la tarea de resistencia a la velocidad demuestra, al igual que pasa con la variable duración, que es significativamente más corta que el resto, salvo transiciones. Esto se debe a la búsqueda de máxima intensidad en el menor tiempo posible. No manifiesta diferencias significativas con transiciones ya que es una tarea también con tendencia a altas intensidades en tiempos cortos.

Por otro lado, las tareas de juego real también obtienen diferencias significativas con las tareas de transiciones y $4 \times 4$. El motivo por el cual se reflejan estos datos es porque el juego real busca la mayor transferencia a la competición, con duraciones largas. Sin embargo, las tareas de transiciones y $4 \times 4$ tienen menor tiempo de intervención porque duran menos.

Teniendo en cuenta los datos expuestos anteriormente, y observando que las diferencias significativas entre las diferentes tareas son distintas en función de cada variable, no hemos podido realizar una categorización, ya que no hay un patrón consistente en el cual podamos basarnos para hacer agrupaciones de tareas.

Es decir, si hacemos una revisión de la TABLA 1, donde se encuentran los datos de todas las tareas en función de cada una de las variables, y de la TABLA 2, donde se encuentran las diferencias significativas entre tareas en función de cada una de las variables, no podemos definir que haya tareas de mayor carga condicional que otras, unas que duren más que otras, etc. Las únicas diferencias importantes que se han conseguido demostrar son entre la tarea de juego real,

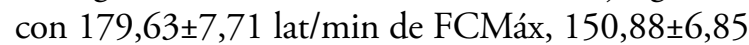

lat $/ \mathrm{min}$ de FCMedia y $6,11 \pm 2,32 \mathrm{mmol} / \mathrm{L}$ de ácido láctico, con datos cercanos a los aportados en otros estudios que obtuvieron una FC Media entre 160 y $170 \mathrm{lat} / \mathrm{min}^{2,13,14,21}$, y con $9 \mathrm{mmol} / \mathrm{L}^{3,20}$, y la tarea de estrategia, con 159,30 \pm 6,45 lat/min de FCMáx, $130,9 \pm 6,1 \mathrm{lat} / \mathrm{min}$ de FCMedia y $2,18 \pm 0,78 \mathrm{mmol} / \mathrm{L}$ de ácido láctico. Estas dos tareas se podrían considerar que pertenecen a dos grupos diferentes, con objetivos condicionales distintos.

Sin embargo, el resto de tipos de tareas no muestra suficientes diferencias entre ellas, como para poder dividirlas en diferentes grupos. Esto puede justificarse, como se observa en la FIGURA 2, con que la gran mayoría de tareas empleadas tienen el mismo perfil a nivel de lactacidemia (por encima del umbral anaeróbico), por tanto, tienen las mismas características fisiológicas, esfuerzos muy similares, con el objetivo de trabajar la resistencia mixta, pero dentro de ellas no se pueden hacer distinciones debido a la gran similitud que presentan.

A partir de los datos obtenidos en el presente estudio, podemos concluir que la diversidad de tareas analizadas es adecuada para el entrenamiento de los diferentes rangos metabólicos que se dan en competición en fútbol sala. Las tareas de juego real en entrenamiento no llegan a alcanzar las características fisiológicas del esfuerzo en competición. Además, las tareas de transición, movilidad, campo completo, $4 \mathrm{x} 4$ y porterojugador presentan características condicionales similares en relación a la FCMáx, FCMedia y concentración de lactato, considerándolas aptas para el desarrollo de la resistencia mixta y umbral anaeróbico. Por otro lado, las tareas de resistencia a la velocidad se adecúan al trabajo de la capacidad láctica. Finalmente, aquellas tareas en las que se trabajan las acciones a balón parado tienen un perfil condicional bajo, pudiendo ser empleadas como tareas regenerativas.

\section{Abstract}

Physiological analysis about futsal training drills

It is important to be able to accurately monitor training load during futsal drills intended for physical development to allow the optimization of training parameters. The aim of this study was to analyze the conditional profile of futsal drills. Eight professional futsal players were assessed for heart rate, blood lactate, duration, and intervention time responses to 8 commonly used futsal training drills. Statistical analysis was realised with SPSS 20.0, and comprises general descriptive statistics and two ANOVA with Bonferroni correction. The results showed that real game exercises not reached the physiological load of matches. Furthermore, speed endurance drills reached bigger lactate concentration than the other futsal 
training activities. Finally, transition, mobility, full field, $4 \times 4$ and fly-goalkeeper drills had similar conditional characteristics, near to mixed endurance and anaerobic threshold. In conclusion, analyzed drills are adequate for the development of the metabolic pathways essential in futsal.

KEYwoRDS: Maxim HR; Medium HR; Lactate; Match; Training.

\section{Referencias}

1. Cano O. Fútbol: entrenamiento global basado en la interpretación del juego. Sevilla: Ed. Wanceulen; 2001.

2. Álvarez Medina J, Giménez Salillas L. Necesidades cardiovasculares y metabólicas del fútbol sala: análisis de competición. Apunts Ed Fís Dep. 2001;67:45-51.

3. Riveiro Rodríguez JE. La preparación física del fútbol sala. Sevilla: Ed. Wanceulen; 2001.

4. Barbero Álvarez JC. Análisis cuantitativo de la dimensión temporal durante la competición en fútbol sala. Eur J Hum Mov. 2003;10:143-63.

5. Barbero Álvarez JC, Soto V, Barbero Álvarez V, Grande Vera J. Match analysis and heart rate of futsal players during competition. J Sports Sci. 2008;26:63-73.

6. Seirulo Vargas F. Opción de planificación en los deportes de largo período de competiciones. RED. 1987;1:53-62.

7. Álvarez Medina J, Corona Virón P, Manonelles Marqueta P. Planificación y cuantificación del entrenamiento en una temporada regular de fútbol sala. Apunts Ed Fís Dep. 2004:76;46-52.

8. Álvarez Medina J, Echávarri JM, López I, Manonelles P, Quílez J, Terreros J. Análisis científico de diferentes métodos de entrenamiento en el fútbol sala. AMD. 2009;28:93-103.

9. De Freitas VH, Miloski B, Bara Filho MG. Quantificação da carga de treinamento através do método percepçáo subjetiva do esforço da sessão e desempenho no futsal. Rev Bras Cineant Des Hum. 2012;14:73-82.

10. Borin JP, Gomes AC, Leite G. Preparação desportiva: aspectos do controle das cargas de treinamento nos jogos coletivos. Rev Educ Fís. 2007;18:97-105.

11. Hopkins WG, Marshall SW, Batterham AM, Hanin J. Progressive statistics for studies in sports medicine and exercise science. Med Sci Sports Exerc. 2009;41:3-13.

12. Castagna C, Barbero Álvarez JC. Physiological demands of an intermittent futsal-oriented high-intensity test. J Strength Cond Res. 2010;24(9):2322-9.

13. Andrín García G. Caracterización de los esfuerzos en fútbol sala basado en el estudio cinemático y fisiológico de la competición. Rev Dig EF Dep. 2004;77.

14. Barbero Álvarez JC, Granda Vera J, Soto Hermoso VM. Análisis de la frecuencia cardíaca durante la competición en jugadores profesionales de fútbol sala. Apunts Ed Fís Dep. 2004;77:71-8.

15. Laia F, Rampinini E, Bangsbo J. High-Intensity Training in Football. Int J Sports Phyisiol Perform. 2009;4:291-306.

16. Freitas V, De Souza EA, Oliveira RS, Pereira LA, Nakamura FY. Efeito de quatro dias consecutivos de jogos sobre a potencia muscular, estresse e recuparaçao percebida, em jogadores de futsal. Rev Bras Educ Fís Esporte. 2014;28(1):23-30.

17. Milanez VF, Ramos SDP, Salle-Neto F, Machado FA, Nakamura FY. Relaçẫo entre métodos de quantificaçao de cargas de treinamento baseados em percepção de esforço e frequencia cardiaca em jogadores jovens de futsal. Rev Bras Educ Fís Esporte. 2012;26(1):17-27.

18. Duarte R, Batalha N, Folgado H, Sampaio J. Effects of exercise duration and number of players in heart rate responses and technical skills during futsal small- sided games. Open Sports Sci J. 2009;2:37-41.

19. Makaje N, Ruangthai R, Arkarapanthu A, Yoopat P. Physiological demands and activity profiles during futsal match play according to competitive level. J Sports Med Phys Fit. 2012;52(4):366-74.

20. Álvarez Medina J, Corona Virón P, Giménez Salillas L, Manonelles Marqueta P. Importancia del VO 2 MÁX. y de la capacidad de recuperación en los deportes de prestación mixta. Caso práctico: fútbol-sala. AMD. 2001;18:577-83.

21. Castagna C, D'Ottavio S, Granda Vera J, Barbero Álvarez JC. Match demands of professional futsal: a case study. J Sci Med Sport. 2008;12:490-4. 
Lago-Fuentes C, et al.

\section{Agradecimientos}

Al club participante en el estudio por todas las facilidades que nos ofrecieron para la realización de la presente investigación. No hubo financiación de ninguna institución ni otro tipo de entidad.

\section{Conflicto de intereses}

Los autores declaran que no hubo conflicto de intereses.

DIRECCIÓN

Carlos Lago-Fuentes

Facultad de Ciencias de la Educación y del Deporte Universidad de Vigo

Campus A Xunqueira s/n 36005 - Vigo - Pontevedra - ESPAÑA e-mail: carloslagofuentes@hotmail.com
Sometido: 27/01/2015

1a. Revisión: 30/o8/2015

2a. Revisión: 07/03/2016

3a. Revisión: 09/04/2016

Aceptado: 15/04/2016 